Case Report

\title{
Impact of Glucose-6-phosphate Dehydrogenase Deficiency on Sickle Cell Anaemia Expression: A Case Report
}

\author{
Patrick Olanrewaju Osho", ", Matilda Adesuwa Osagie Ojo ${ }^{1}$, Evelyn Salewa Osho², \\ Ndidi Aisha Okunnuga ${ }^{3}$, Oluwatosin Idowu $\mathrm{Oni}^{4}$, Olugbenga Festus Fabusiwa ${ }^{5}$ \\ ${ }^{1}$ Department of Haematology \& Immunology, Faculty of Basic Clinical Sciences, University of Medical Sciences, Ondo-City, Nigeria \\ ${ }^{2}$ Department of Radiology, Faculty of Basic Clinical Sciences, University of Medical Sciences, Ondo-City, Nigeria \\ ${ }^{3}$ Oncology Unit, Department of Radiology, Faculty of Basic Clinical Sciences, University of Medical Sciences, Ondo-City, Nigeria \\ ${ }^{4}$ Department of Haematology/Virology, University of Medical Sciences Teaching Hospital, Akure, Nigeria \\ ${ }^{5}$ Department of Medical Services, Ondo State primary Health Care Development Agency, Oke-Eda, Akure
}

Email address:

droshopo@gmail.com (P. O. Osho), matildaojo4@gmail.com (M. A. O. Ojo), esosho@gmail.com (E. S. Osho), aishaokunnu@gmail.com (N. A. Okunnuga), godsprince805@gmail.com (O. I. Oni), drfabusiwa@yahoo.com (O. F. Fabusiwa) ${ }^{*}$ Corresponding author

\section{To cite this article:}

Patrick Olanrewaju Osho, Matilda Adesuwa Osagie Ojo, Evelyn Salewa Osho, Ndidi Aisha Okunnuga, Oluwatosin Idowu Oni, Olugbenga Festus Fabusiwa. Impact of Glucose-6-phosphate Dehydrogenase Deficiency on Sickle Cell Anaemia Expression: A Case Report. International Journal of Genetics and Genomics. Vol. 8, No. 3, 2020, pp. 102-105. doi: 10.11648/j.ijgg.20200803.12

Received: July 16, 2020; Accepted: August 18, 2020; Published: September 21, 2020

\begin{abstract}
Sickle cell anaemia and Glucose-6-phosphate dehydrogenase deficiency have anaemia as major clinical consequence. The two disorders are rarely co-expressed in a patient. However, the pathological co-existence of both disorders tends to worsen and aggravate the clinical presentation in affected individuals. We report a diagnosis of the co-inheritance of these two disorders in a 43 year old man who was diagnosed with SCA at childhood. He was managed in a secondary health care facility since child hood. He has had multiple blood transfusions on account of repeated episodes of haemolytic anaemia which was solely attributed to SCA. He was referenced our center on account of recurrent severe heamolytic anaemia with a PCV of $9 \%$. In the preceding 2 months to detecting his G6PD deficient status, he was having monthly blood transfusions on account of severe anaemia. Following the detection of his G6PD deficiency status and appropriate intervention with glutathione and selenium supplements and counseling to avoid exposure to oxidizing agents, he had a respite in the frequency of acute episodes of haemolysis necessitating blood transfusion, as well as an improvement in his steady state PCV of $16 \%$. This case report underscores the importance of routine screening for G6PD status in patients with SCA so as to institute appropriate measures to reduce the worsening of incidence of acute episodes of haemolysis and the need for recurrent blood transfusions on account of this.
\end{abstract}

Keywords: Glucose-6-phoshate Deficiency, Co-expression, Sickle Cell Disease, Pathological Co-existence

\section{Introduction}

Glucose-6-phosphate dehydrogenase is the rate limiting enzyme in the pentose phosphate pathway that converts glucose-6-phosphate into 6-phosphogluconate [1]. G6PD deficiency is the disorder that results when this enzyme is deficient. G6PD deficiency; the disorder that results when this enzyme is deficient is the most common human enzyme defect which affects more than an estimated 400 million people worldwide [2, 3, 4]. The activity of the pentose phosphate pathway protects red blood cells (RBCs) from oxidative damage by supplying reducing energy to them thus maintaining the level of reduced co-enzyme nicotinamide adenine dinucleotide phosphate (NADPH) [5]. The NADPH 
in turn maintains the supply of reduced glutathione (GSH) in the RBCs which acts like oxidant scavenger that is used to mop up any oxidants (free radicals) that will cause damage to the RBCs [6].

Sickle cell disease (SCD) is a common genetic disorder of haemoglobin in which there is inheritance of mutant haemoglobin genes from both parents [7]. The inheritance of two sickle haemoglobin $(\mathrm{HbSS})$ is referred to as sickle cell anaemia.

Sickle cell disease (SCD) and glucose-6-phosphate dehydrogenase (G6PD) deficiency are inherited chronic haemolytic anaemias prevalent in Nigeria [7]. While SCD is inherited as an autosomal recessive disorder, G6PD deficiency is inherited as a sex-linked recessive genetic disorder that occurs most often in males in heterozygous condition and it occurs in females only in homozygous condition but can be partial in a heterozygous female [7].

In Nigeria the prevalence of sickle cell anaemia is $2-3 \%$ while that of G0PD deficiency is $22 \%[5,9]$. More than 400 million people worldwide are affected by G0PD deficiency [7]. Many studies have showed variation in the prevalence of coinheritance of G6PD with sickle cell anaemia, it ranges between $2-30 \%$. [1, 7, 10, 11]

Glucose-6-phosphate dehydrogenase is expressed in all tissues, where it catalysis the first step in the pentose monophosphate pathway converting glucose- 6 phosphate to 6-phophogluconate with production of reduced nicotinamide adenine dinucleotide phosphate (NADPH). NADPH is used to convert GSSG (oxidized glutathione) to GSH (reduced glutathione) [12]. GSH then reduces peroxides to prevent oxidative damage of cell membrane and other cell constituents Unlike other tissues, erythrocytes depend solely on this pathway for generation of GSH since it lacks mitochondria [12]. Thus erythrocytes are most severely affected by this enzyme deficiency. The co-inheritance of these two disorders has been previously documented $[10,11]$. Its occurrence could result in a more severe clinical course of disease due to exaggerated haemolysis. There is a need to document the clinical course of our patient with co-expression of the two genetic haemolytic disorders, so as to draw the attention to its occurrence as well as the need to have routine screening for G6PD deficiency in our patients with SCA

\section{Case Report}

This is a case of a 43 years old man, a known sickle cell anaemia patient (HbSS) diagnosed at childhood who was referred from general hospital on account of recurrent passage of coca coke coloured urine, jaundice, severe bone pains and generalized body weakness. His stable PCV was said to be $16 \%$, however whenever he had haematuria, his PCV dropped to $9 \%$, this he observed for more than 20 years. Had several hospital admission on account of vaso-occlusion crisis and blood transfusion. Average blood transfusion and hospital admission per year is greater than four $(>4)$. He usually get over the counter analgesics for recurrent bone pains and only present in the hospital when the pain is severe. Have other 2 siblings who have sickle cell anaemia, but they have a milder clinical features and no history of haematuria.

Examination at presentation he was in obvious pain distress, pale, deeply icteric, dehydrated, urine rack showed dark coloured urine. His pulse rate was 135 beat/minute, blood pressure was $130 / 96 \mathrm{mmHg}$, with a $3^{\text {rd }}$ heart sound, respiratory rate was 44 cycles/ minute and tender hepatomegaly of $4 \mathrm{~cm}$. The initial assessment was hyperhemolytic crisis in a known sickle cell anaemia patient.

The complete blood count showed the following: haemoglobin, $3.4 \mathrm{gm} / \mathrm{dL}$, red blood cell $(\mathrm{RBC}) 2.38 \times 10^{12} / \mathrm{L}$, packed cell volume $8 \%$, white cell count (WBC), $13700 / \mu \mathrm{L}$, platelet, $305000 / \mu \mathrm{L}$, absolute reticulocyte count, 0.38 million $/ \mu \mathrm{L}$ (14.3\%) with MCHC $388 \mathrm{~g} / \mathrm{c}$, MPV $10.9 \mathrm{fl}, \mathrm{MCH}$ $32.3 \mathrm{pg}$ and MCV 83.7pg. Blood smear showed anisopoilocytosis, 5 nucleated red blood cell/100 white blood cell, polychromatic cells, many schistocytes, bite cells and blisters cells, leucocyosis with toxic granulations and left shift of neutrophils with thrombocytosis. Direct antiglobulin test was negative. Other laboratory tests showed: serum urea, 4.03 (1.7-9.1 mmol/L), serum creatinine, 148.32 (50-132 U/L) and serum potassium, 3.51 (3-5 $\mathrm{mmol} / \mathrm{L})$. Smear for malaria parasite was negative. Following the result of peripheral smear, the differential diagnosis was made. Heamolytic anaemia secondary to infection and?? G6PD deficiency.

He was resuscitated with IV fluids and intranasal oxygen was commenced. An urgent packed cell volume (PCV) done was $8 \%$ and he had a total of 4 unit of packed cells. He was also commenced on parenteral ceftriaxone, as well as parenteral analgesics. He was discharged home on PCV was $20 \%$, bone pain completely subsided and urine cleared, and placed on his routine medications.

He had G6PD assay done two months later and the result revealed his G6PD deficient status of 231 (244-299) $\mathrm{IU} / \mathrm{mL} /$ Erythrocyte. Further history was taken concerning the G6PD deficient status. There was no family history to suggest G6PD deficiency; none of his siblings with HbSS has been diagnosed to be G6PD deficient. Positive history of use of herbal medications

He was subsequently placed on glutathione supplement and selenium-based therapy along with his routine medications, the frequencies of hemolytic crisis and hospital admission was noticed to have reduced in the last 3 years. His stable PCV ranges between $16-18 \%$

\section{Discussion}

Our patient had features of acute haemolysis with elevated blood creatine kinase levels. G6PD activity in the erythrocytes was very low in spite of the significant reticulocytosis, G6PD activity of the muscle cells correlates well with that of erythrocytes [12]. Thus one may expect that in G6PD deficient individuals there may be symptoms due to oxidative damage of myocytes. Fasola et al. (2019) found higher prevalence of G6PD deficiency in SCD patients compared with controls and the severity of G6PD deficiency was more pronounced in SCD patients than controls. SCD patients were twice more 
likely to have G6PD enzyme activities below $3.0 \mathrm{IU} / \mathrm{gHb}$ than controls [11]. They also noted that G6PD deficient patients had higher levels of absolute reticulocyte counts and the different fractions of bilirubin than G6PD deficient HbAA controls which was also observed in our patient. G6PD deficiency was detected in $35.83 \%$ of the SCD patients with majority of the participants having genotype SS, most of the G6PD deficiencies were found in the participants with genotype SC. In Ibadan, Nigeria, G6PD deficiency was detected in 16 out of 100 participants with SCD [11].

A study of nine G6PD deficient volunteers and nine non-deficient controls after exercise found no increase in blood oxidative biomarkers (reduced glutathione, oxidised glutathione, lipid hydroperoxides, etc.) [12]. This may be partly due to the fact that muscles unlike erythrocytes have alternative way to generate GSH. NADP+ (oxidised form of $\mathrm{NADP}$ ) can be generated by NAD+ kinase, then reduced to NADPH by the action of malic enzyme or/and NADP+ dependent isocitrate dehydrogenase [13]. This alternate pathway may also apply to the oxidative stress situation such as in our patient, but in this instance reactive oxygen radicals may have been too overwhelming for the alternative pathway to compensate totally.

Studies have noted that the coinheritance of G6PD with sickle cell anaemia did not affect the clinical severity of the disease $[11,14]$. It also did confer any biological advantage on the disorder comparing the clinical and laboratory profile of SCD patients with G6PD deficiency and SCD patients with normal G6PD activity showed no statistical difference between the two groups in most parameters except in the haematocrit [10]. In contrast, comparing the blood transfusion rates for both deficient and non-deficient $\mathrm{HbS}$ patients, Benkerrou et al. reported a higher frequency of blood transfusion. [15] This suggests the increased requirement for blood transfusion as seen in our patient. When the two problems coexist, particular care should, therefore, be exercised in the administration of drugs such as antimalarials known to trigger hemolysis in patients with G6PD deficiency. The impact of local herbs in triggering haemolysis in G6PD deficient patient should also be considered.

\section{Conclusion}

SCD patients with G6PD deficiency run the significant risk of developing haemolytic crises, sepsis, haemoglobinuria, and kidney failure after taking anti-malarial drugs or eating oxidative foods like fava beans [16]. Co-existence of the two disorders can worsen the degree of anaemia and thus impact an increase need for blood transfusion. Routine screening for G6PD deficiency should be done in all patients newly diagnosed with SCD. This is important to guide the rational use of drugs used in the management, so as not to precipitate or worsen an ongoing haemolytic process. There need for counseling of G6PD deficient SCD patients so as to avoid oxidative stress from substances with such potentials is also emphasized.

\section{References}

[1] Antwi-Baffour, S., Adjei, J. K., Forson, P. O., Akakpo, S. Kyeremeh, R. and Seidu, M. A. (2019). Comorbidity of Glucose-6-Phosphate Dehydrogenase Deficiency and Sickle Cell Disease Exert Significant Effect on RBC Indices Hindawi Anemia. 2019: 1-9 https://doi.org/10.1155/2019/3179173.

[2] L. van den Broek, E. Heylen, and M. van den Akker, "Glucose-6-phosphate dehydrogenase deficiency: not exclusively in males," Clinical Case Reports, vol. 4, no. 12, pp. 1135-1137, 2016. View at: Publisher Site | Google Scholar.

[3] Cappellini, M. D. and Fiorelli G. 2008. Glucose - 6 phosphate dehydrogenase deficiency. Lancet 371: 64-74. [PubMed] [Google Scholar].

[4] Ronquist, G., and Theodorsson E.. 2007. Inherited, non spherocytic haemolysis due to deficiency of glucose - 6 phosphate dehydrogenase. Scand. J. Clin. Lab. Invest. 67: 105111. [PubMed] [Google Scholar].

[5] Manju T (2017) Glucose 6 phosphatase dehydrogenase (G6PD) and neurodegenerative disorders: Mapping diagnostic and therapeutic opportunities. Genes Dis. 4 (4): 196-203.

[6] Prchal JT, Gregg XT. Red Cell Enzymes. Hematology/ASH Education Book. Available at http://asheducationbook.hematologylibrary.org/content/2005/1 /19.full?sid=5fb34610-c164-4deb-9e85-0922086efaea. Accessed: June 29, 2020.

[7] Ogunkanbi, S. O, Adedoyin, O. T., Biliaminu, S. A. and Ernest S. K. (2019) Prevalence of Glucose-6- Phosphate Dehydrogenase Deficiency among Steady-State Sickle Cell Disease Children at University of Ilorin Teaching Hospital, Ilorin. Open Journal of Pediatrics, 9, 29-38.

[8] Umar M. Sickle cell anaemia: Prevention and control. 2010 http://www.gamji.com/article5000/NEW5314.HTM. Accessed July 28 th 2020 .

[9] Jidda M L, Ibrahim KK, Aiki G, Ngaski AA, Blessing J, et al. (2017) The Prevalence of Glucose-6-Phosphate Dehydrogenase (G6PD) Deficiency in Students of Sultan Abdurrahaman School of Health Technology Gwadabawa, Sokoto, North-Western Nigeria. International Blood Research \& Reviews. 7 (4): 1-6.

[10] Firempong CK, Nsiah K, Yeboah FA. (2016). Some clinical and haematological effects of G6PD deficiency among individuals with sickle cell disorders in Kumasi, Ghana. The Journal of Medical Research. 2 (3): 81-85.

[11] Fasola, F. A., Fowodu, F. O., Shokunbi, W. A. and Kotila, T. R. (2019). The effect of the coinheritance of glucose-6-phosphate dehydrogenase deficiency on the severity of sickle cell disease. Niger Postgrad Med J; 26: 118-22.

[12] Kalliopi G, Ioannis GF, Dimitrios D, Konstantinos P, Panagiotis T, et al. (2019) Exercise in Glucose-6-Phosphate Dehydrogenase Deficiency: Harmful or Harmless? A Narrative Review Oxidative medicine and cellular longevity 2019 (6): $1-7$.

[13] Yang Y, Sauve A A. (2016) NAD (+) Metabolism: Bioenergetics, Signaling and Manipulation for Therapy. 1864 (12): 1787-1800. 
[14] Belisario A R, Rodrigues S R, Elvin T N, Velloso R, Maria S C, et al. (2016) Glucose -6-Phosphate dehyrogenase Deficiency in Brazillian Children With Sickle Cell Anaemia is not Associated With Clinical Ischaemic Stroke or High-Risk Transcranial Doppler. Padiatr Blood Cancer. 63: 1046-1049.

[15] Benkerrou M., Alberti C., Couque N., Haouari Z., Ba A. et al. (2013). Impact of glucose-6-phosphate dehydrogenase deficiency on sickle cell anaemia expression in infancy and early childhood: A prospective study. Br J Haematol. 163: 646-54.

[16] Samuel Antwi-Baffour, Jonathan Kofi Adjei, Peter Owadee Forson, Stephen Akakpo, Ransford Kyeremeh and Mahmood Abdulai Seidu (2019). Comorbidity of Glucose-6-Phosphate Dehydrogenase Deficiency and Sickle Cell Disease Exert Significant Effect on RBC Indices. Hindawi Anemia. https://doi.org/10.1155/2019/317917. 